Gesnerus 54 (1997) 251-257

Short Communication

\title{
Zum rätselhaften Bild des Kosmos bei Camille Flammarion
}

Daniel Lukas Bäschlin

\section{Summary}

In 1888 the French astronomer, Camille Flammarion, published in one of his popular books a picture of a man breaking through the confinement of the old cosmos. This picture has been reproduced frequently and has served as an attractive illustration for many purposes ever since. There was a long debate on the origin of this picture: does there exist an original Renaissance source of it or is it a creation of Flammarion or of one of his illustrators?

This Paper introduces a new and so far unrecognized element into this discussion. The ancient greek philosopher, Archytas, is said to have asked the question: is it possible for a man to stretch his hand or his stick out of the edge of the cosmos? The picture of Flammarion seems to be a precise and almost literal illustration of this question.

\section{Zusammenfassung}

Der französische Astronom Camille Flammarion publizierte 1888 in einem seiner populärwissenschaftlichen Bücher ein Bild, das einen die Grenze des antiken Kosmos durchbrechenden Menschen zeigt. Dieses Bild wurde in der letzten Zeit oft reproduziert und fand als attraktive Illustration eine vielfältige Verwendung. Der Ursprung dieses Bildes ist nach wie vor unklar: Gibt es ein Renaissance-Original oder ist das Bild erst von Flammarion oder einem seiner Illustratoren geschaffen worden?

Dr. D. Bäschlin, Via Panigari 8, CH-6600 Locarno 
Die vorliegende Arbeit bringt ein neues, bisher übersehenes Element in diese Diskussion. Der griechische Philosoph Archytas soll die Frage gestellt haben: Kann man am Rande des Kosmos die Hand oder den Stock hinausstrecken? Das Bild bei Flammarion scheint eine genaue, fast wörtliche Illustration dieser Frage zu sein.

In unserem Jahrhundert, besonders in den letzten Jahrzehnten, ist im Bereich der Naturwissenschaft und ihrer Geschichte ein Holzschnitt von fast magisch wirkender Faszination als Illustration in vielen Büchern und Zeitschriften in Erscheinung getreten (Abb.). Er zeigt einen Menschen, den Weltrand durchstossend, in eine unbekannte, unerwartete Weltweite jenseits der Sterne hinausblickend. Woher und aus welcher Zeit stammt dieser Holzschnitt? Wer ist der Holzschneider? Alle bisherigen Nachforschungen sind ergebnislos geblieben! Man konnte dieses Welt-Bild bis jetzt nicht weiter zurückverfolgen als bis zu einem Buch des französischen Astronomen Camille Flammarion ${ }^{1}, 1888$ in Paris herausgekommen, des Titels «L'Atmosphère, météorologie populaire».

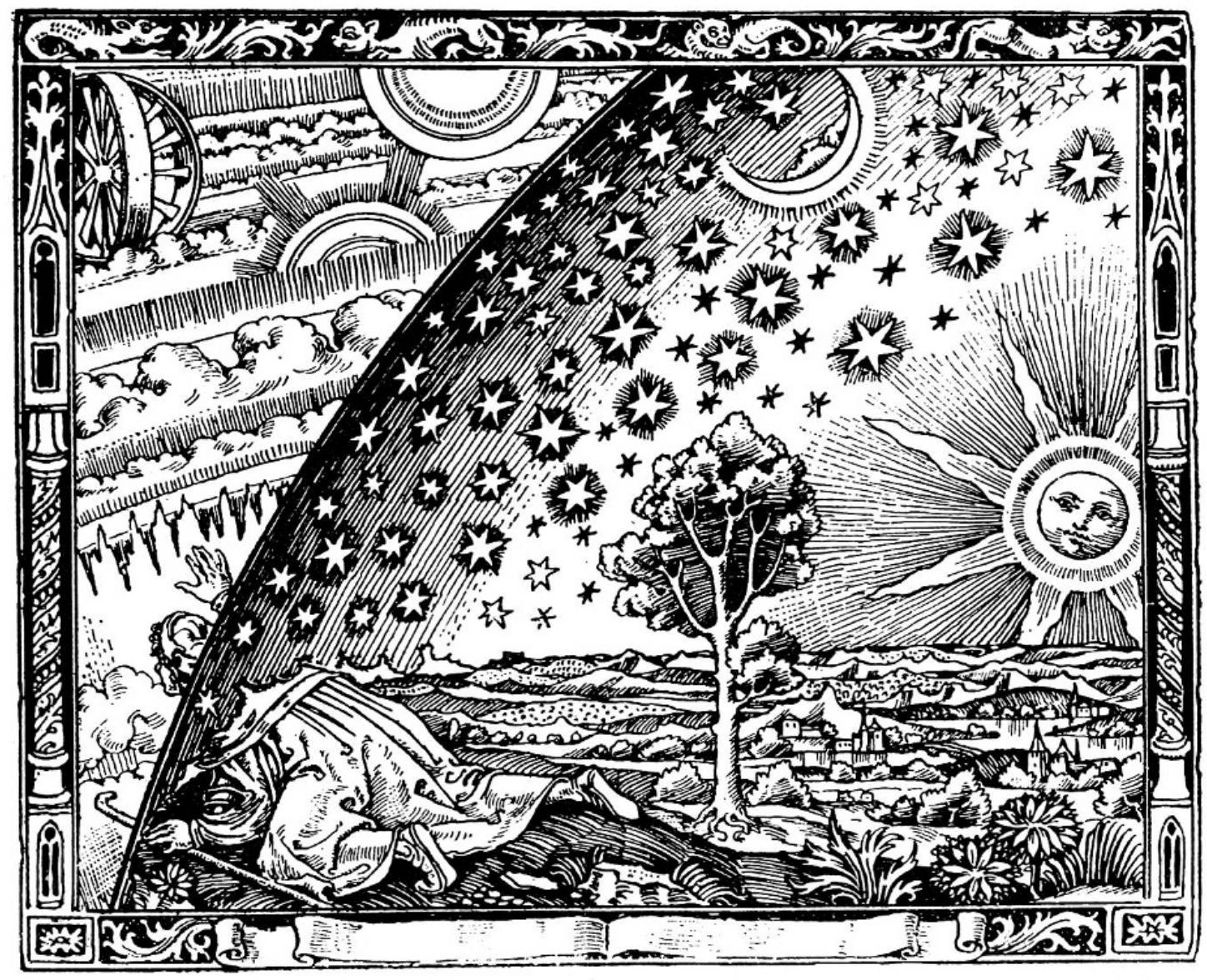

«Un missionaire du moyen âge raconte qu'il avait trouvé le point où le ciel et la Terre se touchent ...», in: Camille Flammarion, L'Atmosphère, météorologie populaire, Paris 1888, S. 163. 
Bruno Weber ${ }^{2}$ hat im Gutenberg-Jahrbuch von 1973 einen umfangreichen Artikel zu diesem Holzschnitt veröffentlicht und die These aufgestellt, Flammarion selbst sei der Holzschneider dieses Welt-Bildes gewesen. Ein grosses Verdienst dieses Artikels ist die vollständige Zusammenstellung aller Publikationen, die sich seit 1888 mit diesem Holzschnitt befassten, sowie die vielseitigen Nachforschungen, die Weber eigenständig zu ihm anstellt. Die These, er stamme von Flammarion selbst, ist auf Grund der Weberschen Argumentation eine Möglichkeit, aber keineswegs bewiesen. Die im folgenden vorgetragene Untersuchung soll veranlassen, die Suche nach einem Ursprung in der Renaissance nicht vorschnell abzubrechen. Wir werden zweierlei zu unterscheiden haben: Interpretation und Herkunft.

\section{Interpretation}

\section{a) Vermutungen}

Die Vielfalt der Interpretationen spiegelt die Faszination, die von diesem Holzschnitt ausgeht. Hier einige markante Beispiele aus dem Artikel von Weber ${ }^{3}$ :

«Mittelalterliche phantastische Darstellung des Weltsystems» (Foerster, 1903).

«Der Zweifler, der mit seinem Kopf das Kristallgewölbe durchbricht» (Bürgel 1903).

«Cette naïve représentation rapelle la conception de Thales» (Urbain/Boll, 1932).

Das Bild zeigt «die Kühnheit des Nikolaus von Cusa... der forschende Mensch, als Wanderer dargestellt, durchbricht die äussere unveränderliche Schale des Fixsternhimmels ... und erblickt draussen neue Räume» (Dessauer, 1948).

«Es gibt einen Holzschnitt des 16. Jahrhunderts, der bezeichnend für die Neugier dieser Zeit zu sein scheint» (Zinner, 1957).

«...aus dem gläubigen Christen wurde ein rechnender und staunender Pantheist» (Würtenberger, 1957).

«Eine etwa aus dem Jahre 1552 stammende, naive Darstellung zeigt sehr anschaulich die ungeheure Wandlung des Weltbildes, welche der Gedankengang des Cusanus bedeutet. Wir sehen einen Wanderer am 〈Erdrande〉 angekommen, er hat die Fixsternschale durchbrochen und Kopf und rechte Hand durch die Lücke gestreckt: staunend erhebt er sie, denn er blickt in eine endlose Welt ganz fremder, fremdartiger kosmischer Gebilde» (Walter Kranz, 1957 - wie gezeigt werden soll, hätte gerade er, Mitarbeiter an Diels «Fragmente der Vorsokratiker», etwas anderes dazu sagen können).

«Dieses Bild aus dem XVII. Jahrhundert, welches vermutlich eine Rosenkreuzerische Erleuchtung darstellt...»: «Der Pilger, der offenbar auf einer 〈pélérinage de l'âme〉 begriffen ist, bricht durch die nächtliche Grenze seiner Welt...» (C.G. Jung, 1958).

«Der Mensch durchbricht den endlichen Kosmos des Mittelalter» (Meschkowski, 1961).

1 Camille Flammarion, L'Atmosphère, météorologie populaire, Paris 1888, S. 163. Die Illustration trägt den Titel «Un missionaire du moyen âge raconte qu'il avait trouvé le point où le ciel et la Terre se touchent...» und wird auf der vorangehenden Seite kurz kommentiert.

2 Bruno Weber, Ubi caelum terrae se coniungit. Ein altertümlicher Aufriss des Weltgebäudes von Camille Flammarion. In: Gutenberg-Jahrbuch 1973, S. 381-408.

3 Bruno Weber, a.a.O., S. 399ff. 
Diese letzte Formulierung wird dann - bis heute - zu einer festen Formel, die von Corti (1961), Lüst (1964), Sänger-Bredt (1964), von der Osten-Sacken (1965), Becker (1968), Kohne (1968), Hemleben (1969), Berninger (1972) aufgenommen und später in Schulbüchern der Geschichte und Physik wiederholt wird.

«Sehnsucht nach dem Unendlichen». Unter diesen Titel wird das Bild von Aniela Jaffé, einer Schülerin C.G. Jungs, gestellt. Sie fragte weitherum vergeblich nach seiner Herkunft. Das Warburg-Institut in London teilte ihr mit, nach Ansicht seiner Experten stamme der Holzschnitt aus dem 19. Jahrhundert. Andererseits stellt sie die Stilverwandtschaft mit Hans Weiditz dem Jüngeren fest (geb. vor 1500, gest. um 1536), der als Holzschneider und Buchillustrator tätig war.

\section{b) Die Evidenz im Lichte des Archytas}

Die Suche nach dem Sinn dieses Holzschnittes soll auf eine ganz andere Spur gelenkt werden, die zu einem konkreteren Ergebnis führen dürfte. Es ist uns überliefert, dass der Philosoph, Mathematiker und Staatsmann Archytas von Tarent (ein Freund und Zeitgenosse Platons) die Frage gestellt hat ${ }^{4}$ :

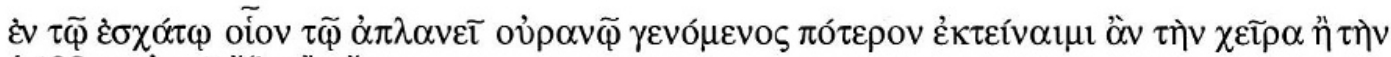

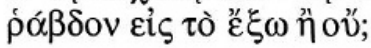

«Kann ich, wenn ich mich am Rand des Himmels, d.h. in der Sphäre der Fixsterne, befinde, diesen Rand mit der Hand oder dem Stock durchstossen, oder kann ich das nicht?»

Diese Frage trifft einen kritischen Punkt. Denn wenn es, wie Aristoteles formuliert ${ }^{5}$, ausserhalb des Kosmos nicht einmal leeren Raum gibt, dann kann der Kosmosrand weder mit Stock noch Hand noch sonst etwas durchstossen werden.

Unser Holzschnitt zeigt nun mit überraschender Augenfälligkeit: Die drei Kennzeichen «Hand», «Stock» und «Durchstossung des Kosmosrandes» sind so unverkennbar und offensichtlich, dass unzweifelhaft erscheint: Dieser Holzschnitt ist eine Illustration der Archytas-Frage!

Noch ein anderes Bildelement des betrachteten Holzschnitts passt vorzüglich zur Archytas-Interpretation: In der christlichen Tradition gibt es von früh her unzählige Darstellungen eines Jenseits. Aber diese unterscheiden sich von der Jenseitsdarstellung unseres Holzschnitts in wesentlicher Hinsicht: Sie stellen die Ikonographie der religiösen Himmelswelt dar, erfüllt von Gott,Christus, Heiligem Geist, Maria, Heerscharen von Engeln und symbo-

4 Hermann Diels, Die Fragmente der Vorsokratiker, 6. Aufl. hg. v. Walther Kranz, Berlin 1951, Bd. 1, S. 430, A 24.

5 Aristoteles, Peri ouranou 279a 12-14. 
lischen Tierwesen. Nichts dergleichen auf unserem Holzschnitt. Im Gegenteil: Alles Anthropomorphe - Sonne und Mond als Menschenwesen - befindet sich innerhalb der Fixsternsphäre. Man hat zwar die Räder des Ezechiel bemüht, die gelegentlich so gedeutet werden wie auf unserem Bild:senkrecht zueinander zu einem Doppelrad verflochten. Aber bei Ezechiel gehören diese Räder zum Gottesthron und ihre Felgen sind voller Augen - sie sind völlig eingebettet in religiöse Ikonographie ${ }^{6}$. Im Gegensatz dazu mutet das Jenseits unseres Holzschnitts unheimlich an durch fremdartige Gebilde ohne das geringste Lebenszeichen. Es erscheint durchaus als Versuch, so etwas wie anaximandrische oder demokritische Welten jenseits der unseren darzustellen ${ }^{7}$ Kirchlich-theologisch gesehen gehört, was hier als Jenseits sichtbar wird, zur Natur, zur Schöpfung, nicht zu einer göttlichen Sphäre, die, als Bereich des Schöpfers, von aller Natur radikal unterschieden vorgestellt wird. Es ist auch klar, in welchem Sinn die Kirche zur Archytas-Frage Stellung genommen hat. Augustin verwirft die anaximandrische Lehre ${ }^{8}$, und Thomas von Aquin bekennt sich zur einen, von Gott begrenzten und geführten Schöpfungswelt ${ }^{9}$. Die Jenseitsvision unseres Holzschnitts ist also in bezug auf kirchliche Überlieferung revolutionär, ketzerisch, gefährlich. Es war zweifellos riskant, diesen Holzschnitt zu publizieren.

$\mathrm{Zu}$ den im vorigen Abschnitt aufgeführten Interpretationen ist folglich zu sagen: Der Mensch, der hier abgebildet ist, ist weder ein Wanderer noch ein Pilger, noch ein Neugieriger, erst recht kein Rosenkreuzer oder Seelenwanderer oder Unendlichkeitssehnsüchtiger, sondern Archytas, dem der Holzschneider auf seine Frage gleichsam - ganz im Sinne etwa des Giordano Bruno - zuruft: «Ja, du kannst!»

Weber behauptet: «Das Bild... bleibt naturwissenschaftlich unbestimmbar, ist als Rebus (= Bilderrätsel) nicht zu lösen.» ${ }^{10}$ Er dürfte kaum recht gehabt haben. Jedenfalls erfolgte diese Behauptung in Unkenntnis der Archytas-Frage.

6 Ez. 1, 15-22.

7 Hermann Diels, a.a.O., Anaximandros A17.10ff. In bezug auf den Holzschnitt ist auch das S. 84, Z. 9ff. Erwähnte sehr reizvoll: Anaximander nahm an, die Fixsterne seien ein Lichtdurchbruch von jenseits der Fixsternsphäre her, was das Feuer im Jenseitsbereich des Holzschnitts erklären würde.

8 Augustin, Civitas Dei VIII 2.

9 Thomas von Aquin, Summa theologica, Romae 1925, Teil I, Quaestio 47, Articulus 3. (I, S. 384) «... Unde necesse est, quod omnia ad unum mundum pertineant. Et ideo illi potuerunt ponere plures mundos, qui causam mundi non posuerunt aliquam sapientiam ordinantem, sed casum; ut Democritus, qui dixit, ex concursu atomorum factum esse hunc mundum, et alios infinitos. AD PRIMUM ergo dicendum, quod haec ratio est, quare mundus est unus, quia debent omnia esse ordinata uno ordine, et ad unum. Propter quod Arist. in 12, Metaph., ex unitate exemplaris probat unitatem mundi, quasi exemplati.»

10 Bruno Weber, a.a.O., S. 398. 


\section{Herkunft}

Die Archytas-Frage ist der Nachwelt durch den Neuplatoniker Simplicius überliefert, und zwar in dessen Kommentar zur Aristotelischen «Physik», der 1526 in Venedig neu herausgegeben wurde ${ }^{11}$. Diese Herausgabe schien ein geradezu feierlicher Akt gewesen zu sein, zu dem sich die Regierung der Stadt entschieden hatte. Die Überlieferung der Archytas-Frage und mit derselben das Problem, ob die Schöpfung endlich oder unendlich sei, hat Denker der frühen Renaissance in Anspruch genommen, besonders Nikolaus von Kues (1401-1464), der erstmals den Gedanken äusserte, der unendliche Gott habe auch eine unendliche Welt geschaffen. Mit besonderer Wucht tritt diese Problematik wenig später bei Giordano Bruno (1548-1600) in Erscheinung, der zum ersten Mal den für seine Zeit unerhörten Gedanken ausspricht, die Fixsterne seien insgesamt Sonnen wie die unsere, nur viel weiter entfernt. Unserem Holzschnitt käme danach, vom Inhalt her gesehen, geistesgeschichtlich eine hervorragende Bedeutung zu.

Es waren vor allem die Florentiner des 15. Jahrhunderts, die den Neuplatonismus wieder entdeckten und folglich Simplicius lasen, wobei sich - was erst in jüngster Zeit genügend berücksichtigt wurde - gerade auch die berühmten Maler dieser Zeit davon beeinflussen liessen ${ }^{12}$. Ihr neuartiges Kunstschaffen strahlte nach Deutschland aus, indem sich bedeutende deutsche Meister teils an Ort und Stelle von der florentinischen Renaissance inspirieren liessen. Weber zufolge galt der Holzschnitt des Flammarion nach ersten Nachforschungen als deutsches Erzeugnis aus der ersten Hälfte des 16. Jahrhunderts: «Dies bestätigen die Kunsthistoriker Röttinger, einer der besten Kenner des altdeutschen Buchholzschnitts, 1931, und Würtenberger 1957, mit einem Ansatz 〈zwischen 1530 und 1550〉 bzw. 〈um 1550〉.» ${ }^{13}$ Dies passt gut zum Erscheinungsjahr des Simplicius-Kommentars. Auf Grund unserer Archytas-Interpretation neigen wir dazu, diese Datierung nach wie vor ernsthaft in Erwägung zu ziehen.

Wenn diese Archytas-Interpretation zutrifft, gibt es einen Gesichtspunkt, von dem aus die Urheberschaft Flammarions unwahrscheinlich erscheint: Nehmen wir an, Flammarion (oder einer seiner Mitarbeiter) habe den Holzschnitt selbst hergestellt, so kann er nicht aus einer Kenntnis der ArchytasFrage und einer Absicht, diese zu illustrieren, entstanden sein. Denn sonst hätte Flammarion dies in seinem Kommentar erwähnt. Statt dessen kom-

11 Ueberweg-Heinze, Geschichte der Philosophie, Berlin 1909, Band I, S. 349. Diels hat den Kommentar 1882 neu herausgegeben.

12 Edgar Wind, Heidnische Mysterien in der Renaissance, Suhrkamp 1984.

13 Bruno Weber, a.a.O., S. 384. 
mentiert er den Holzschnitt in einem ganz anderen Zusammenhang, der mit dem Archytas-Probblem nicht das geringste zu tun hat ${ }^{14}$. Hat er ihn jedoch ohne Kenntnis der Archytas-Frage hergestellt, so ist es höchst unwahrscheinlich, dass seine Bildkonzeption zufällig perfekt zu gerade dieser Frage passt wie der Schlüssel ins Schloss. Wenn aber der Holzschnitt, den Flammarion publiziert, auf der Vorlage einer bereits bestehenden Buchillustration beruht, so ist in diesem unbekannten Buch der ursprüngliche Sinn des Holzschnitts bereits vergessen, und Flammarion übernimmt dessen bereits sinnfremden Kommentar oder legt sich selber einen solchen zurecht.

14 Flammarion, L'Atmosphère, S. 162. 